Voix et Images

\title{
Percer le mur du son du sens, une entrevue avec Madeleine Gagnon
}

\section{Lucie Robert et Ruth Major}

Volume 8, numéro 1, automne 1982

Madeleine Gagnon

URI : https://id.erudit.org/iderudit/200361ar

DOI : https://doi.org/10.7202/200361ar

Aller au sommaire du numéro

Éditeur(s)

Les Presses de l'Université du Québec

\section{ISSN}

0318-9201 (imprimé)

1705-933X (numérique)

Découvrir la revue

Citer ce document

Robert, L. \& Major, R. (1982). Percer le mur du son du sens, une entrevue avec Madeleine Gagnon. Voix et Images, 8(1), 5-21. https://doi.org/10.7202/200361ar d'utilisation que vous pouvez consulter en ligne.

https://apropos.erudit.org/fr/usagers/politique-dutilisation/ 


\title{
Percer le mur du son du sens, une entrevue avec Madeleine Gagnon
}

\author{
par Lucie Robert, Université Laval \\ et Ruth Major, Université du Québec à Montréal
}

V.I. Vous avez fait une thèse de doctorat sur Paul Claudel en 1968, pourquoi?

M.G. Déjà, en 68, j'étais très loin de ma thèse dans la mesure où je l'ai soutenue cette année-là. C'est un projet qui datait de 62 . J'avais été très marquée par la poésie symboliste. Je voulais faire une étude narratologique qu'on appelait à l'époque une analyse structurale. Je voulais tenter l'analyse complète d'un très long texte, les Cinq grandes odes, parce que ca me semblait, même si cette idée peut paraître paradoxale, beaucoup plus hermétique, plus difficile d'accès que la poésie de Rimbaud. Il faut dire que j'aimais autant l'écriture poétique de Claudel que son écriture dramatique alors plus connue. La lecture de cette œuvre a beaucoup marqué mon écriture. Très brièvement, je dirais qu'il y avait dans son écriture une réflexion, un art poétique qui était tissé dans sa propre écriture. C'est ce qui m'intéressait et $\mathrm{m}$ 'a beaucoup intéressée par la suite. J'ai toujours voulu ne pas dissocier les deux versants de l'écriture: le versant réflexif et le versant fictif.

V.I. L'année 68 a été une année trouble: il y a eu les événements de mai en France, la résistance à la guerre du Vietnam aux États-Unis, le mouvement du retour à la terre... est-ce que cela a influencé l'écriture des Morts-vivants ou est-ce un projet personnel qui était arrivé à son terme?

M.G. Je dirais que cela a influencé peut-être de façon extérieure. Il y a des marques de ces événements dans les Morts-vivants, plus particulièrement de la guerre du Vietnam. Ce sont presque des marques de tatouage sur l'écriture. Pour le reste, le retour à la terre, à la nature, ça ne m'a jamais marquée; peut-être parce que je n'avais pas besoin d'y retourner. C'est de là que je viens. C'est présent dans mon œuvre mais je n'ai jamais été influencée par la mode ou la pensée du retour à la terre de cette époque-là. Je n'ai jamais été contre non plus.

V.I. Vous êtes venue à l'écriture relativement tard. Est-ce qu'il y a une raison à cela? 
M.G. Je ne me sentais certainement pas prête à risquer publiquement une première œuvre. En ce qui concerne la première que j'ai risquée autour de vingt-huit ans, les Morts-vivants, quand je la relis, je me dis que je ne l'aurais pas lancée comme ca. Je ne me considérais pas prête pour toutes sortes de raisons. Ce n'est pas que je n'avais pas de textes et même plusieurs - j'écris depuis que j'ai quatorze ans à peu près mais je ne sentais pas qu'il y avait une œuvre mûrie. J'avais besoin de poursuivre une réflexion. Je faisais des études de maîtrise en philosophie. Après, j'ai fait le doctorat en lettres. Je sentais le besoin de continuer à lire, à réfléchir, à analyser, suivant mon œuvre à moi, l'accompagnant, la préparant dans ses mutations.

V.I. L'expérience de Chroniques et des Herbes rouges a été très importante, pouvez-vous nous en parler?

M.G. Ça a été très important. Je mettrais ces expériences - celles de Chroniques, des Herbes rouges et même celle de I'U.Q.A.M. d'une certaine façon puisqu'elle a coïncidé avec les deux autres - sous le paradigme de la solidarité. C'est une époque où on sentait énormément le besoin de travailler en groupe, dans des lieux définis, circonscrits, où on pouvait mettre en rapport des recherches théoriques, des questionnements philosophiques qui nous permettaient de penser l'écriture autrement, y compris dans ses formes. C'étaient des lieux où il n'y avait pas de censure sur les recherches théoriques, issues par exemple de la psychanalyse, des sciences humaines, de l'histoire des mouvements sociaux, y compris du mouvement féministe. Ces lieux voulaient lier une pratique artistique et une pratique politique. Ça a été très important pour moi d'y participer, de sortir d'une espèce de solitude d'écrivain - ça se disait beaucoup à l'époque. Entre nous, on avait l'impression d'être moins seuls...

V.I. Est-ce que votre départ de Chroniques et des Herbes rouges et votre rattachement à VLB est lié à un événement spécial ou s'il s'agit simplement d'un passage?

M.G. Non. Je trouve la question délicate et je voudrais y répondre de façon nuancée parce qu'il y a beaucoup de choses qui ont été écrites par certains écrivains des Herbes rouges qui ont perçu notre départ - celui de Philippe Haeck et le mien - comme une espèce de trahison. C'est dommage. J'ai beaucoup de respect pour les frères Hébert et pour la majorité des écrivains des Herbes rouges. Je crois que si j’ai quitté c'est parce qu'il était impossible de le faire dans la mesure où, sans être un groupe structuré et défini politiquement, c'était quand même un groupe relativement homogène, avec beaucoup de cohésion. Finalement, au fil des années, la solidarité - qui me semblait nécessaire au début s'est traduite pour moi par des virtualités censurantes. Je ne dis pas qu'il y avait de la censure aux Herbes rouges, je dis que j'ai pressenti l'impossibilité de pratiquer une écriture dans un groupe qui était trop identifié, trop fermé. Ça se traduisait par un certain esprit de chapelle 
ou de secte. Non pas que les Herbes rouges aient manipulé ouvertement un tel état d'esprit mais ça n'est pas innocent non plus que nos passages ailleurs aient été traités de trahisons. C'est un peu comme si on avait délaissé un groupe politique. On n'avait pas le droit de quitter les Herbes rouges et c'est ce qui m'a poussée à le faire: pour retrouver l'autonomie et la solitude essentielles à l'écrivain. Il est possible que d'autres auteurs des Herbes rouges n'aient pas vécu à ce point ce fantasme du collectif. II y avait aussi une autre raison: je ne suis pas certaine que les Herbes rouges ait été intéressée à reprendre la publication de mon œuvre depuis ses débuts. Je ne crois pas que les Morts-vivants aurait fait l'affaire des Herbes rouges. Je n'ai pas pratiqué un seul type d'écriture, une écriture. II n'y a pas uniquement dans mon œuvre (ce qui correspond très bien à ce que peut représenter l'écriture des Herbes rouges), ce qu'on a appelé l'écriture de la modernité. Les Morts-vivants, et d'une certaine façon Lueur, échappent à ces modalités formelles en devenir. Ce sont des textes du futur antérieur. Je les assume et ne veux absolument pas les renier.

V.I. Est-ce que vous pensez que le milieu intellectuel est un milieu privilégié pour le féminisme?

M.G. Je ne le sais pas parce que je ne fais pas partie d'un autre milieu. Qu'est-ce qu'un intellectuel? C'est quelqu'un qui sait penser et qui sait exprimer sa pensée par la parole ou l'écriture. Je dirais que, pour le mouvement féministe, c'est un privilège de pouvoir expliquer avec sa parole une aliénation historique, une subordination des femmes, dans toutes ses nuances. C'est un privilège mais je ne crois pas qu'il appartienne au milieu intellectuel. J'ai vu des gens incapables de s'exprimer sur les rapports intersexuels hommes-femmes, ou hommes-hommes, femmes-femmes, qui font pourtant partie du milieu intellectuel. J'en connais d'autres qui ne font pas partie du milieu intellectuel et pour qui ces questions ont été très présentes et très vivantes. Pour tout projet de libération, l'idéal consisterait à ce que nous devenions tous et toutes des intellectuels. L'anti-intellectualisme ambiant au Québec $m$ 'effraie. Vous remarquerez qu'il n'est prôné que par des intellectuels qui se renient. C'est un grand danger que de renoncer aux privilèges des choses de l'intelligence.

V.I. Considérons tout de même le milieu intellectuel au sens le plus large: quels sont, à votre avis, les effets du féminisme sur ce milieu depuis une douzaine d'années, peut-on remarquer des changements, une évolution?

M.G. Oui, je crois qu'il y a eu d'énormes changements partout. II y a eu des changements qu'on peut moins mesurer, inventorier, circonscrire, dans les rapports intimes, amoureux entre les hommes et les femmes, entre les femmes, entre les hommes. II y a certainement, grâce aux voix de femmes et aux choses inédites et inouies qu'elles ont signifiées, une nouvelle intelligence amoureuse manifeste. Par ailleurs, il y a aussi des changements visibles dans tous les milieux de travail; il y a des 
femmes pour qui ces transformations ont donné lieu à une véritable prise de conscience. D'une certaine façon aussi, toutes les sciences - et surtout les sciences humaines - sont bousculées par cet éveil récent des femmes. La biologie, la sexologie, la psychologie, l'ethnologie, la science politique ne peuvent plus ignorer les postulats sexistes qui les fondaient en grande partie. L'histoire et la philosophie sont elles aussi fondamentalement bouleversées. Les pratiques de recherche et d'enseignement ont dû tenir compte de ces questions lancées d'abord par les femmes sous forme de cris. La psychanalyse a aussi été fortement ébranlée dans ses assises par les questions des femmes. Par exemple, la théorie de la castration, centrale en psychanalyse, est fondamentalement remise en question par les recherches des femmes analystes ou analysantes. On voit là une révolution, un bouleversement. Ca s'est produit aussi pour l'écriture. On assiste depuis le début des années 70, à une nouvelle révolution de la pensée, de l'écriture. Le saut, qualitatif, est du même ordre que les grands bouleversements apportés par des mouvements comme le surréalisme ou l'automatisme. Il serait trop long ici de nommer tous ces bouleversements mais je crois que, dans tous les domaines de la pensée, l'éveil des femmes a été marquant. Les bouleversements conceptuels et formels apportés par la voix des femmes en mouvement et en éveil constituent sans doute la plus grande révolution intellectuelle des temps modernes. C'est sans doute pour cette intelligence originale que nous sommes attaquées par des intellectuels qui se renient.

V.I. Comment voyez-vous votre apport au féminisme dans le cadre d'un milieu d'écriture et de recherche?

M.G. Je le vois différemment que je le voyais. Avec la naissance du mouvement des femmes, je voyais mon écriture emportée par ce mouvement, de sorte qu'il n'y avait plus de distinction entre lutte politique de libération et écriture. Ça s'est manifesté entre autres dans des textes comme la Venue à l'écriture, ou Retailles, ou encore Pour les femmes et tous les autres. D'une certaine façon, ce sont des textes militants, des textes dont l'écriture prend en charge certaines questions politiques. Maintenant, comme plusieurs autres, je crois que les femmes écrivains ont été libérées petit à petit de l'obligation de se prendre pour les porte-parole d'un mouvement qui, de toute façon, nous dépasse. On assiste à un retour à l'écriture et à l'exploration de l'écriture qui n'est plus nécessairement tributaire d'une volonté de transformer les rapports politiques. Là encore, j'ai senti un certain danger ces dernières années de sectarisation causée probablement par la nécessité de la mise sur pied d'organisations politiques dont l'écriture des femmes devrait rendre compte. L'écriture des femmes devrait prendre en charge, selon une certaine morale sectaire, les finalités politiques du mouvement. Je crois que c'est très dangereux et que ça peut donner lieu à des censures qui ne seraient pas acceptables parce qu'elles viendraient des femmes que celles qu'on a déjà connues avant: censure des expérimentations formelles, censure de l'activité d'écrire qui doit être absolument libre, 
autonome. On ne peut dicter, à cause de principes politiques, les visées de l'expérimentation formelle, tributaire de lois ayant justement échappé aux censures du refoulement: celles de l'inconscient et de ses représentations pulsionnelles et imaginaires. Le rêve, la fiction ne se programment pas. J'ai pris mes distances par rapport à l'organisation politique, à toute organisation politique.

V.I. Comment voyez-vous le rôle et la fonction de critique alors?

M.G. Je vois difficilement parce que la critique est devenue tellement importante. Elle est devenue une superstructure qui, à la limite, peut fonctionner sans les textes. Actuellement, la critique académique domine toute la critique, pas seulement la critique universitaire. Ces dernières années, il existe une espèce d'hypertrophie du métalangage, de finalité scientifique de la critique. Je trouve bizarre que la critique se mette à fonctionner toute seule comme un satellite qui tournerait indéfiniment dans l'espace, sur lui-même, branché sur rien. Je crois à la nécessité d'un retour à la lecture des textes. Au «lire-écrire», comme dit Roger Laporte. La critique doit redevenir une lecture et la finalité de toute lecture devrait donner une autre écriture et non pas un modèle d'analyse. Toute lecture immanente découvre un modèle original compris dans chaque texte. Pour lire véritablement des textes, il faut beaucoup d'humilité. II faudrait que la critique renonce à. ses finalités scientifiques et accepte le fait que le texte ne sera jamais réductible par aucun outil scientifique, quel qu'il soit. Elle devrait renoncer à ses prétentions scientifiques et redevenir un art. Ou alors, qu'elle s'assume autonome et se déclare elle-même nouvelle science linguistique: qu'elle avoue donc qu'elle n'a rien à voir avec les écritures ou peu de choses. Les écritures de fiction lui serviraient en quelque sorte de pré-texte, en vue de l'élaboration d'un objet qui pourrait se passer du sujet textuel. II faut bien le dire, aucune science de la subjectivité n'est possible. Le sujet n'est que paragramme mouvant. C'est dans cette fluxion que naît l'écriture. La lecture est un renoncement en acte à la clarification de ce qui vit de l'obscurité. D'une certaine façon, l'analyse du texte, comme toute analyse subjective, ne peut être que spectrale. Avec la psychanalyse, nous savons que les spectres de l'histoire - les blancs de mémoire - sont tout aussi signifiants que la parole pleine. La lecture est, tout autant que l'écriture, l'assomption du vide et de l'absence. Cela ne s'assigne pas à résidence académique. Je n'ai envie de censurer aucune recherche scientifique; ce qu'il faudrait dire c'est qu'il n'y a plus d'adéquation entre la critique et le texte, de façon générale, actuellement.

V.I. Est-ce qu'il y a une évolution de la critique littéraire, vis-à-vis de l'écriture des femmes particulièrement?

M.G. Je crois qu'on peut le remarquer. D'abord, au tout début, on voyait que les critiques ne voulaient absolument pas tenir compte du phénomène qu'on a appelé "l'écriture des femmes", "l'écriture de la féminité" ou "l'écriture féminine». Ils manifestaient une résistance très grande, 
affichée la plupart du temps par un refus de lire les textes de femmes autour des années 70 . On disait qu'il s'agissait d'un mouvement politique et que ça n'avait rien à voir avec l'écriture de la modernité. On assiste à un changement dans la mesure où maintenant la critique tient compte de ce mouvement comme d'un mouvement d'écriture. L'expression «écriture de la féminité " a fait son chemin. La critique, de façon générale, est prête à admettre que de ce côté-là, il se passe quelque chose de nouveau du point de vue de l'écriture qu'on dit d'avant-garde - je n'aime pas cette expression que Barthes qualifiait, à juste titre, de «métaphore militaire». On parle même maintenant, dans les revues savantes, de "l'écrire-femme». On est passé un peu trop brutalement d'une occultation quasi totale à une trop grande valorisation - qui est un effet de mode - et on assiste à un autre type de censure aujourd'hui. Rares sont les critiques, hommes ou femmes, qui vont oser critiquer, lire, se permettre de lire une œuvre écrite par une femme, surtout si cette œuvre a des prétentions féministes. A partir du moment où on se dit féministe, on peut écrire n'importe quoi. S'il n'y a pas, de la part des critiques, une très grande vigilance, on risque de se faire emporter, une autre fois, par un phénomène qu'on avait déjà identifié avec le socialisme - quand on parlait de réalisme socialiste. A ce propos, on pourrait parler de l'émergence d'un nouveau réalisme, un réalisme féministe, un réalisme politique qui viendrait donner ses ordres aux textes, en ordonner le déroulement, les finalités, les visées sociales. Je trouvé que c'est un danger. Ce serait le rôle de la critique, justement, de «lire-écrire» les écritures politiques, les manifestes politiques féministes qui empêchent les explorations individuelles de l'écriture.

V.I. Et vos textes, comment la critique les a-t-elle accueillis?

M.G. II y a un écrivain qui disait: «ll est beaucoup plus facile de trouver un écrivain que de trouver un bon lecteur», je ne sais pas qui disait cela, mais je trouve que c'est vrai. II y a très peu de bons lecteurs. Pour moi, un critique devrait être un lecteur. Malheureusement, ce n'est pas toujours le cas. La majorité des lecteurs de textes ne font pas de critique et la majorité des critiques ne font pas de véritable lecture: ils s'amènent avec leurs grilles et n'acceptent pas d'entrer dans l'écriture d'une ceuvre. Une œuvre, ca ne s'explique pas et si oui, l'explication ne peut venir que d'elle-même. C'est dans l'œuvre qu'il faut trouver les réseaux d'analyse. Chaque texte véritable de fiction comporte en lui-même les outils, les matériaux de sa propre analyse. J'ai pris conscience que les lectures les plus intelligentes venaient d'écrivains qui s'adonnent à la lecture. Ayant sans doute concrètement, dans leur propre écriture, assumé leur capacité de déroute, la fiction de l'autre semble mieux les toucher. Toute lecture, comme toute écriture, est une perte de soi et de l'autre. Un abandon des préjugés et des a priori, un échec à la transcendance.

V.I. Vous avez enseigné à I'U.Q.A.M., est-ce que les cours que vous avez donnés, les ateliers que vous avez animés vous ont apporté quelque chose? 
M.G. Beaucoup. L'enseignement est un métier qui, en plus de m'avoir permis de vivre, - parce qu'on ne vit pas de l'écriture au Québec quand on écrit ce que j'écris - est dans une très grande proximité avec le métier d'écrivain. Il me permettait de poursuivre ma réflexion sur l'écriture et c'était essentiel de la poursuivre tout en écrivant. Et qu'est-ce que l'enseignement de la littérature sinon une réflexion fondamentale sur l'écriture? Ça nourrissait ma pratique d'écriture. Je sentais aussi ma pratique d'enseignement nourrie par mon métier d'écrivaine. Les deux pratiques n'étaient pas vécues de façon opposée ou contradictoire: elles s'éclairaient l'une et l'autre.

V.I. Le rapport entre votre écriture et celle de vos étudiants a dû se ressentir alors comme une manière d'échange, de discussion?

M.G. Oui, les deux. C'est un rapport de réciprocité. J'ai connu plusieurs étudiants, de véritables écrivains, qui ont publié. Plusieurs sont maintenant connus: François Charron, Sylvie Gagné, Mireille Lanctôt, Danielle Fournier. J'en ai connu d'autres qui seront publiés probablement, peutêtre pas, mais qui sont des écrivains. Ils venaient suivre mes cours, en tous cas, mes ateliers d'écriture, pas seulement parce que j'étais enseignante et que j'animais les ateliers, mais aussi parce que j'étais écrivaine. Souvent, ces jeunes avaient une connaissance intime de mon œuvre. C'était très enrichissant pour moi. J'ai toujours été très consciente des limites de mon écriture, de là où ça devait s'arrêter pour les étudiants. Souvent le travail des ateliers consistait en une prise de conscience de leur propre capacité d'écriture. Il fallait alors analyser les transferts qui pouvaient s'opérer sur mon écriture. C'était intéressant parce que, par l'analyse du transfert, les étudiants découvraient leur propre force, leurs capacités individuelles d'écrivain. J'étais toujours heureuse quand un étudiant ou une étudiante se découvrait dans le cadre des ateliers ou même des cours. J'y ai toujours permis les travaux de théorie ou de critique littéraire autant que les travaux de fiction favorisant une prise de conscience de leur capacité respective: leur permettre de découvrir s'ils étaient plus doués, plus motivés pour la recherche théorique, pour la recherche critique ou encore pour l'écriture de la fiction? C'était toujours des moments très heureux pour moi quand un étudiant ou une étudiante se réconciliait avec ses forces, qu'elles soient théoriques, critiques ou du côté de la fiction.

V.I. Pourquoi quittez-vous l'enseignement, particulièrement l'enseignement à I'U.Q.A.M.?

M.G. J'ai parfois l'impression que je pourrai répondre à cela dans dix ans, quand j'aurai pris une distance par rapport à cette décision toute récente, une grande décision, je le sais. C'est pas I'U.O.A.M. que je quitte, en fait, c'est une institution. Je crois que j'aurais quitté toute institution littéraire, universitaire, scientifique, à ce stade de ma vie. Là où j'en étais dans ma recherche d'écriture, il me fallait absolument quitter l'institution de façon régulière. Je ne quitte pas l'enseignement, j'aimerais beaucoup retour- 
ner, donner des cours de temps en temps dans les universités ou dans les cégeps. Donner des cours, être en contact avec la génération des jeunes qui apprend, qui expérimente. J'aimerais aussi lire des mémoires et des thèses, diriger encore des travaux d'écriture. Je ne quitte donc pas l'enseignement, je ne quitte pas I'U.Q.A.M. non plus, je quitte une institution littéraire scientifique. Je quitte la stabilité du discours parce que c'est irréconciliable avec ma pratique d'écriture. C'est Edmond Jabès qui dit dans son dernier livre d'entretiens, Du désert au livre: «L'écriture commence là où la parole se termine». Pour parler, il faut être très structuré, organisé; pour écrire, il faut être dans la désorganisation, la déstructuration, la déroute totale. L'activité d'enseignement, de façon régulière, est stable, sécuritaire et sécurisante, elle oblige constamment à une structuration de la pensée pour pouvoir passer à la parole. J'éprouvais le besoin de quitter le champ du discours organisteur et structuré sur le texte littéraire. Ce qui ne veut pas dire que je ne ferai plus de théorie ou de critique, mais je crois que je le ferai autrement, dans beaucoup plus d'indéterminé et d'indéfini. L'enseignement nous oblige à beaucoup de détermination.

\section{V.I. Quels sont vos projets futurs?}

M.G. J'ai plein de projets, comme tout le monde. Des beaux projets: de vivre, d'aimer, de voyager, d'essayer de me débrouiller pour ne pas avoir trop de difficultés financièrement. J'ai deux ou trois projets d'écriture dont un, commencé, qui est une espèce d'essai de l'écriture sur l'écriture. Un essai sur l'écriture se faisant, qui tente de travailler les limites institutionnelles. C'est là que je vais essayer de creuser les limites génétiques de l'écriture aux deux pôles opposés de sa sphère. Du côté de la naissance impossible de l'écriture et du côté de l'institutionnel, de sa mort possible. C'est un projet plus théorique, où la fiction coule aussi. Je ne veux pas de différence ou d'opposition entre l'écriture théorique et l'écriture de fiction. Je voudrais travailler la réflexion théorique en n'annulant pas le surgissement de la fiction dans le processus de l'écriture. Je travaille aussi à un tout petit recueil de poèmes: des versets très brefs, un livre "le plus mince possible», selon l'expression d'André Beaudet dans I'Expectative de la nuit des temps. II y a aussi un projet très vaste en train de frayer sa voie: il s'agit d'un récit élaboré, structuré, à la limite de la linéarité contiguë.

V.I. Pourquoi avez-vous choisi l'écriture comme pulsion privilégiée?

M.G. L'écriture n'est pas une pulsion, elle est générée par la pulsion, comme toute recherche, qu'elle soit scientifique ou artistique. Pourquoi l'écriture? Je ne le sais trop. J'ai beaucoup hésité quand j'étais jeune. C'était l'expression artistique, de façon générale, qui m'intéressait: j'hésitais entre le piano, le théâtre, même la peinture. Pourquoi l'écriture? C'est sans doute parce que l'écriture utilise les mots et que j'aime beaucoup les mots, les "vocables", pour parler comme Jabès. Dans l'univers des mots, on est très près de l'univers de la pensée. J'ai toujours 
été extrêmement attirée par le monde philosophique et quand j'ai choisi l'écriture - parce que j'ai choisi d'être écrivaine - c'est la proximité avec le monde de la pensée, de la réflexion philosophique qui m'attirait tout particulièrement. L'écriture a le don d'explorer la folie artistique, d'utiliser les mots de tous les jours et aussi d'utiliser les mots de la pensée et de la réflexion philosophique.

V.I. Quelle est l'importance du dessin dans cela? Dans Au cœur de la lettre, il y a des encres que vous avez faites, est-ce que ça va prendre plus d'importance dans votre œuvre?

M.G. Oui, j'espère. J'ai toujours fait des encres. Il y a une double importance. Ces encres-là accompagnaient un mouvement d'écriture: ce ne sont pas des encres qui illustrent $A u$ coeur de la lettre. Elles sont le geste pictural qui accompagnait le geste de l'écriture. Ça s'est fait ensemble, en même temps, dans un grand rapport amoureux entre les deux modes d'expression. Je tenais à les reproduire aussi parce que, comme pour l'écriture, c'était un geste. J'assumais d'une certaine façon mon innocence en acceptant de laisser imprimer des encres qui n'étaient pas celles d'une artiste qui a suivi des cours de dessin. J'ajouterai ceci : pour Lueur, j'avais demandé à un graveur-poète que j'aimais beaucoup de faire des illustrations. Il avait lu Lueur et m'avait dit au téléphone: «je n'ai rien vu». Ça m'avait beaucoup blessée. Je regrettais ma demande. Alors, j'ai écrit dans Au cœur de la lettre: «Le poète-graveur n'avait pas vu mon écriture, c'est possible". Je me suis dit: "Tiens, pourquoi je ne mettrais pas mes encres à moi ?". C'est drôle parce que dans le temps, je recevais une lettre d'une amie belge, poète-philosophe, Françoise Delcarte, - qui est un peu connue ici - qui me demandait certaines de mes encres qu'elle avait déjà vues parce qu'elle voulait absolument écrire à partir d'elles. Ça m'a un peu consolée du premier refus. J'ai trouvé intéressant qu'une écrivaine soit stimulée par mes dessins alors qu'un graveur n'avait rien vu dans mes poèmes. J'ai trouvé le paradoxe intrigant.

V.I. Vos livres paraissent à intervalles réguliers; est-ce l'indice d'une écriture à peu près continue ou si vous pratiquez l'écriture périodique?

M.G. J'écris tout le temps. Je ne passe pas tant d'heures à ma table d'écriture mais j'y écris tous les jours, soit le journal, soit des lettres, soit dans mon agenda. J'aime écrire: des notes de cours, des petites réflexions, des petits bouts de poèmes; dans des grands cahiers, dans des cahiers plus petits. J'ai un petit cahier qui s'appelle "le petit livre des rêves", j'ai un grand cahier qui s'appelle "le grand livre des pensées». J'écris tout le temps mais jamais d'une façon ordonnée. Ça s'ordonne après coup: à un moment donné, je vois un projet de livre. C'est la phase finale d'un projet de livre qui me fait travailler de façon constante et régulière à une table, à une machine à écrire. Autrement, ça fait partie de ma vie.

V.I. Est-ce qu'il y a un décor, une ambiance particulière qui est nécessaire à l'élaboration d'un projet? 
M.G. Chez moi. Parce que c'est surtout chez moi que j'écris, dans la maison que j'aime, autour des objets que j'aime, entourée des personnes que j'aime, dans l'ouverture. Je suis incapable d'avoir « une Chambre à soi», pour parler comme Virginia Woolf - j'ai une table d'étude, mais je n'écris jamais dessus: ça m'énerve comme ça m'énervait d'écrire dans les bibliothèques. Le plus souvent, j'écris partout dans la maison, surtout sur la table de la salle à manger, entourée de beaucoup de mouvements doux: il faut que je sente les êtres autour de moi. La musique aussi est très importante. Ce sont des musiques différentes selon les périodes. Depuis deux ans, j'écoute surtout de la musique classique, les opéras, les voix, les oratorios, les chceurs. J'aime beaucoup écrire avec des voix ces temps-ci. La musique transforme mon temps, les voix m'animent.

V.I. Est-ce que vous faites une distinction entre la prose et la poésie?

M.G. On en a tellement fait que je répéterais des clichés probablement. Si je pense aux Morts-vivants et à Lueur, je constate que c'est par la prose qu'il m'est donné de traduire une certaine continuité. La poésie me permet de saisir le "fractionnement de l'instant", pour dire comme René Char. Jakobson avait déjà, dans les Essais de linguistique générale, proposé une distinction que je nuancerais, mais qui mapparaissait quand même intéressante: il plaçait la prose du côté de la contiguïté, de la continuité et la poésie du côté de la métaphore, de l'éclatement, de la simultanéité. C'est vrai d'une certaine façon. Mais il y a des moments de l'écriture de la modernité, de mon écriture, où on se demande si c'est du roman ou de la poésie. Avec Antre et Lueur, on ne sait pas trop; avec Au cœur de la lettre non plus. C'est avec les symbolistes qu'on a commencé à parler de poèmes en prose pour traduire cette écriture qui dépasse les frontières des genres. De toute façon, dans mon écriture, comme dans celle de plusieurs autres de mes contemporains, les barricades des genres n'éclatent pas tellement entre prose et poésie, mais entre théorie et fiction.

V.I. Finalement, tout est conciliable?

M.G. Tout. Je pense, oui. Dans toute recherche d'écriture véritable, on doit pouvoir concilier ce qu'on veut concilier.

V.I. Pour revenir à la censure, est-ce que, à la rigueur, ca ne pourrait pas devenir un mode de production ou est-ce que la censure ou le manque de censure ne pourrait pas aussi conduire à l'anéantissement du texte, à son éclatement?

M.G. Je ne comprends pas. Un mode de production, ca veut dire quoi? J'aime bien réserver les termes à leurs champs respectifs. Pour moi, le mode de production renvoie à l'univers de l'économie. Ces emprunts à l'économie politique furent aussi une mode des années 70 . Cela s'est manifesté au groupe Tel Quel, mais aussi ailleurs, de sorte qu'on plaquait un peu partout la théorie de la valeur. Tout devenait réductible - le corps, l'esprit, le texte, l'inconscient - au monde de l'industrie et des machines, 
les signes linguistiques étant eux-mêmes des marchandises en état de production et de circulation.

V.I. Alors, posons la question autrement: est-ce que la censure est simplement une barrière ou si, au contraire, elle ne provoque pas des types d'écriture?

M.G. Je vais essayer de comprendre par ce que j'ai vécu. C'est vrai : il y a deux sortes de censures pour une écriture, l'extérieure et l'interne, celle qu'on appelle "l'auto-censure", les deux jouant constamment. J'ai découvert en réfléchissant à des censures qui me sont venues de l'extérieur, qu'il y avait quelque chose dans mon œuvre qui était de l'ordre de l'autocensure, qui avait provoqué, qui avait prêté le flanc, à la censure extérieure. J'ai été amenée à y réfléchir très profondément. Une fois, une revue très connue avait refusé l'un de mes textes sous prétexte qu'il n'était pas à la hauteur de mon écriture antérieure. J'ai tenté d'analyser ce jugement: comment me voyait-on, comment me fantasmait-on, qu'est-ce que je représentais, qu'est-ce qu'on voulait que je dise? (Je signale en passant que le texte refusé était d'ailleurs la première partie d'Au cœur de la lettrel. Donc, je m'étais posé des questions: qu'est-ce qui, dans mon écriture, provoque des effets de censure? Grâce à cet événement, j'ai été conduite à mieux comprendre des éléments d'autocensure. Je ne sais pas si ça répond bien à la question, mais j'ai compris qu'il y avait des choses dans mon œuvre antérieure qui avaient contribué à fabriquer une représentation de l'œuvre, récupérée d'une certaine façon, renvoyée autrement que ce pour quoi je l'avais lancée. Ce phénomène est d'ailleurs très bien expliqué dans la Leçon de Roland Barthes à l'occasion d'une réflexion sur l'œuvre de Pasolini. Je crois important de citer Barthes à ce sujet:

Se déplacer peut donc vouloir dire: se porter là où on ne vous attend pas, ou encore et plus radicalement, abjurer ce qu'on a écrit (mais non forcément ce qu'on a pensé), lorsque le pouvoir grégaire l'utilise et l'asservit. Pasolini a été ainsi amené à abjurer (le mot est de lui) ses trois films de la Trilogie de la vie, parce qu'il a constaté que le pouvoir les utilisait - sans cependant regretter de les avoir écrits:

"Je pense, dit-il dans un texte posthume, qu'avant l'action, on ne doit jamais, en aucun cas, craindre une annexion de la part du pouvoir et de sa culture. II faut se comporter comme si cette dangereuse éventualité n'existait pas... Mais je pense aussi qu'après, il faut savoir se rendre compte à quel point on a été utilisé, éventuellement, par le pouvoir. Et alors, si notre sincérité ou notre nécessité ont été asservies et manipulées, je pense qu'il faut absolument avoir le courage d'abjurer."

Ces réflexions de Pasolini, entérinées par Barthes, je les assume à mon tour.

C'est ce que je pourrais dire de plus juste sur la censure.

V.I. Vous parlez souvent de l'histoire hystérique, qu'est-ce que c'est? 
M.G. Je ne savais pas que j'en parlais souvent. C'est un terme synthétique, un jeu de mots qui est revenu dans plusieurs textes de femmes autour des années 75. Ce qu'on voulait dire ou souligner fut remarquablement illustré par Hélène Cixous quand elle a ressuscité Dora, qu'elle a réécrit son portrait autrement que Freud ne l'avait fait. Elle est partie d'un des personnages hystériques les plus connus de l'histoire de la psychanalyse pour le comprendre et le faire 'vivre d'une toute autre façon. Dans son Portrait de Dora, elle outrepasse en quelque sorte la barrière du symbolique - et du rapport œdipien au père sur lequel Freud avait buté - pour entrer dans cette zone imaginaire du rapport fille-mère. Le corps hystérique peut alors sortir de sa gangue douloureuse. Nous retrouvons le même phénomène de conversion heureuse dans le très beau livre de Marie Cardinal, les Mots pour le dire, et dans plusieurs autres livres de femmes ces dernières années. Ces femmes ont réussi, à partir de leurs symptômes hystériques, soit grâce à la psychanalyse, ou grâce à elles, à leur écriture, à faire elles-mêmes une conversion du symptôme hystérique douloureux, du corps souffrant, en symptômes, en métaphores d'écriture, en création poétique. C'est Lacan qui dit que la métaphore est un symptôme. C'est très simple à comprendre. Le sang qui coule ou la paralysie qui peut s'installer dans le corps d'une femme sont des métaphores blanches, greffées au corps, des surplus de paroles vides, des taches de mutisme, des lettres aphones. À partir du moment où on prend conscience que ce sont des symptômes de malheur historique, dans les rapports intersexuels hommes-femmes, à partir du moment où les femmes prennent conscience de cette histoire et du fait que l'hystérie n'est qu'un effet de cette histoire d'oppression et d'assujettissement, les symptômes hystériques de douleur peuvent devenir de très belles choses, être transformés en figures de création, en figures poétiques, en écriture. Quand on ne peut pas parler, quand on n'a pas le droit à la parole, sur la place publique, la parole politique, la parole littéraire, quand on n'a pas de parole pour s'expliquer qui on est comme sujet d'histoire, ce sont des symptômes de toutes sortes qui vont se mettre à parler, à témoigner de cette absence. Les femmes ont toujours excellé à faire parler leur corps - il y a de très belles choses à ce sujet dans Parole de femme d'Annie Leclerc. C'est d'ailleurs pour ça qu'on retrouve à profusion ce thème dans les écrits récents des femmes. $\mathrm{Ce}$ phénomène a échappé à la majorité critique qui s'est offusquée et a dit en quelque sorte: " $y$ en a marre de l'écriture du corps des femmes, on en a assez, on est tanné». Ça fait des siècles, des générations que les symptômes corporels ont parlé et on voudrait laver tout ça en cinq ans de prise de parole. Ça ne va pas durer cinq ans, ça va durer un temps infini ; il faudrait peut-être que les critiques s'y préparent. Les femmes continueront de parler de leur histoire dans laquelle le corps de jouissance et de souffrance est très présent.

V.I. Pour une époque où les femmes n'avaient pas d'autre parole que celle-là, ce serait un peu l'équivalent de la recherche scientifique que les hommes faisaient? 
M.G. Oui. L'hystérie était une façon de dire: "Je souffre, donc j'existe.»C'est la meilleure protection contre la folie. Tous les symptômes douloureux peuvent être convertis en immenses bonheurs corporels. L'écriture, de façon toute singulière et inédite, peut suivre les traces de ces conversions. Les femmes ont toujours été très près de leur corps et c'est peut-être pour cela que maintenant, dans leurs textes, on retrouve beaucoup de ce qu'on appelle "la parole du corps". Il y a plein de tatouages sur le corps des femmes qui doivent s'écrire, sinon, ils vont faire très mal. S'écrire ou se parler autrement. Ils peuvent se parler ailleurs que dans l'écriture, mais ils peuvent aussi se parler dans l'écriture.

V.I. Pour vous, la folie est-elle un signe de conscience ou un moteur de connaissance, de recherche, d'engagement, quelque chose qui mène à l'écriture?

M.G. Je crois que la folie est un très grand malheur. C'est d'abord une souffrance. II y a assurément une folie douce, qu'on peut appeler folie blanche, celle qui n'est pas «absence d'œuvre", pour parler comme Foucault, la belle folie de la marginalité, de la création artistique, de la prise de parole excentrique, de la capacité de dire ce qui n'est pas prévu par la norme, ce qui n'est pas convenu, la capacité de dire ou d'exprimer dans diverses formes artistiques l'inconvenu, l'inconnu. Mais il y a aussi la folie très malheureuse qui est la psychose. Quand on a connu des psychotiques, on ne peut pas trouver que c'est une force révolutionnaire. On témoignerait alors d'un manque d'empathie. La folie "absence d'œuvre" est une grande souffrance, elle comporte beaucoup de douleurs. Ce n'est pas vrai que les schizophrènes sont heureux. Ceux qui en reviennent nous disent tous les moments très malheureux de cet étrange voyage intérieur. A ce sujet, il existe de très beaux textes de poètes, je pense à Antonin Artaud, à Emma Santos, par exemple. Je trouve touchante et courageuse la lucide dérive de leurs écritures. Là encore, je crois que lorsqu'elle s'assume, la folie peut devenir une force positive. Par elle peuvent advenir des entendements nouveaux.

V.I. À la lecture de vos textes, on a l'impression d'assister à l'éclatement des verbes, à l'éclatement du je en particulier, des pronoms, des personnes: est-ce que cette dissolution ne viendrait pas de la capacité de nommer les choses, mais pas d'agir sur elles ou sur les gens?

M.G. Si l'écriture ne transforme pas ses lecteurs en scripteurs ou créateurs virtuels, ce n'est pas la peine d'écrire. Si un texte ne conduit pas au moins un lecteur vers une mutation radicale, ça n'est pas de l'écriture. Ces réflexions sont finement élaborées par Mallarmé et Blanchot. Par Borduas et François Charron aussi. Ça ne signifie pas que l'écriture puisse agir sur le réel. L'écriture ne retrouve jamais le réel. Elle doit retrouver la vérité d'une histoire, la vérité des histoires, mais jamais la réalité. L'écriture a lieu là où ça n'a plus de prise sur la réalité des choses. Quand elle écrit les choses, les nomme ou les décrit, ce sont déjà des objets 
construits. S'il y a un tel éclatement des genres, des verbes et de leurs temps, des personnes de la conjugaison, une telle dissolution, c'est justement parce que l'écriture ne travaille pas avec du réel ou avec des choses, mais avec de l'objet construit parce que nommé. Elle travaille, fait vivre l'objet, ce que j'appelle «l'être-de-choses». L'écriture fait vivre des "êtres-de-choses" en leur donnant une dimension d'images, une dimension de figures, qu'anciennement on nommait tropes, une dimension métaphorique, ce que Baudelaire appelait "l'étincelle créatrice de la métaphore", termes repris par Lacan dans I"Instance de la lettre. Quand on se situe à ce niveau, on est dans l'éclatement de tout, de toutes les normes, y compris des normes linguistiques. II n'y a plus de verbes, il n'y a plus de pronoms, il n'y a plus de temps qui tiennent, ils se tiennent tous ensemble dans leur dissolution respective, dans leur organisation interne réciproque.

V.I. Ça conférerait aux choses et aux gens nommés une sorte de passivité?

M.G. Ces «êtres-de-choses» n'ont absolument aucune autonomie. Ils peuvent renvoyer à des personnages ou à des événements réels, ils ne sont pas une traduction, mais une approximation fugace et très nuancée. Ils n'ont pas d'autonomie, ni absolue, ni relative. C'est peut-être pour ça que je suis absolument contre l'expérience de la psychocritique. Elle assume constamment l'être vivant, la chose vivante, l'être réel, la chose réelle qui serait cette latence de l'écrit. L'écriture ne repose sur aucune garantie, ce qui ne veut pas dire qu'elle n'est pas responsable, en toute subjectivité, de l'histoire dont elle est issue.

V.I. Dans vos textes, quelle est la fonction du collage, de l'intertextualité?

M.G. II y avait au début la prise de conscience collective, l'espèce de besoin d'exprimer une solidarité dans les textes. J'ai exagéré de ce côté-là avec Pour les femmes et tous les autres et Poélitique. Je le fais beaucoup moins maintenant. C'est devenu une mode ici et toutes les modes m'énervent. Pour moi, ça a toujours été une façon de reconnaître les influences et ceux et celles, parmi les penseurs ou les écrivains, qui m'avaient marquée. C'est une façon de leur dire: "Vous êtes présents, je pense à vous, je vous lis, je vous reconnais." C'est une façon de reconnaître, ça continue de l'être, mais c'est moins marqué qu'avant, justement à cause de l'effet de mode et d'une facilité dans le collage ou le «patchwork». Parfois, c'est aussi une façon de prendre des raccourcis, par rapport à ma propre trajectoire d'écriture, en collant une citation. Il faudrait que je les analyse. Parfois, c'est vraiment un raccourci pour une pensée ou une expérimentation formelle qui n'ose pas aller jusqu'au bout d'elle-même. C'était peut-être aussi une façon de sécuriser, de dire: "Je ne viens pas de nulle part, je viens d'ailleurs, je viens de plusieurs et je ne suis pas seule». Une manière sécuritaire d'écrire. C'est l'aspect négatif. J'accepte beaucoup mieux que l'œuvre soit fissurée, qu'elle soit incertaine. L'aspect positif est la reconnaissance et j'y tiens 
beaucoup. Je crois que je vais continuer de reconnaître qui j'aime parmi les écrivains et les écrivaines.

V.I. Les collages autres que les citations ont-ils une fonction?

M.G. Oui. C'était une époque. On avait l'impression d'être plus forts: "J'ai ma gang avec moi», "Je ne suis pas toute seule». C'est une façon de combler ou de se consoler comme écrivain parce que l'expérience de l'écriture est une expérience d'infinie solitude, qu'elle est désertique et parfois désespérante. C'est vrai qu'il y a beaucoup de plaisir à écrire, mais il faut être très fort pour parvenir, au-delà du désert des mots, à la jouissance. Cette hypertrophie référentielle et intertextuelle était aussi en partie, à l'époque, une manière de se donner bonne conscience scientifique et politique, un air de dire: «Je ne suis pas juste une écrivaine, je sais des choses et plus, je fais partie des masses." C'était toute cette époque-là. J'en suis revenue parce que j'ai découvert, malgré moi et malheureusement, que dans tout collectif, sont mises en place des finalités de pouvoir nuisibles à l'écriture et somme toute, à l'acte créateur. Si on se met à voir et à nommer les structures hiérarchiques aliénantes d'un collectif, on s'aperçoit que cette parole n'a pas sa place. La parole qui dit, qui montre le pouvoir est une parole qui ne peut pas avoir sa place dans un collectif ou dans une organisation, qui doit se dire seule d'une certaine façon. Cette parole, isolée, devient irrecevable.

V.I. Est-ce que votre engagement politique est venu avant ou après l'écriture collective ou si tout s'est fait en même temps?

M.G. Ça s'est fait en même temps. Ça correspondait au même désir de transformation politique. A cette époque, il y avait une espèce d'ébullition politique au Québec. Des écrivains qui voulaient assumer dans leurs œuvres des préoccupations de libération politique fondamentales, d'autres, des femmes écrivains, devenaient en quelque sorte les porteparole du mouvement féministe. Pour ma part, cet engagement s'est manifesté par le milieu syndical ou avec la revue Chroniques, qui se voulait un outil de subversion comme bien d'autres. Ça s'est vécu aussi dans des groupes militants du mouvement féministe. Les livres collectifs écrits avec des femmes en sont les conséquences. Ça répondait au désir commun, à la même volonté de transformer en profondeur les rapports de classes et les rapports intersexuels et ça a été très important pour moi.

V.I. Qu'est-ce qui vous en reste aux niveaux de la recherche et de l'écriture?

M.G. II me reste évidemment de très beaux souvenirs parce qu'on avait l'impression d'avoir le vent dans les voiles. D'autres nous avaient précédés, dans d'autres pays, mais on ne les connaissait pas assez bien, de sorte que, sans nous en apercevoir, on reproduisait des expériences déjà vécues. On pensait qu'on allait changer, par l'écriture, du réel politique. On pensait en plus que, par l'écriture collective, on allait contribuer à ce changement de manière plus radicale. Je crois qu'il y a eu à cette époque-là une espèce de transfert moral, transfert qui n'a jamais 
été analysé. On est passé d'une morale religieuse catholique à une autre qui, elle, était politique. On ne s'apercevait pas qu'on était de plain pied dans la morale avec ses ordonnances inéluctables pour tout ce qui touche la vie, y compris la vie de l'écriture. Finalement, ces commandements s'avéraient aussi néfastes que ceux de la religion catholique. S'il y a eu ce transfert massif, c'est qu'on ne s'était jamais assumés historiquement comme fondamentalement religieux catholiques. On n'a jamais fait ce procès, on a balayé tout ça du jour au lendemain, au début des années 60 et on a fait comme si le problème tant décrié n'existait plus. II y a donc ici tout un refoulé historique à analyser. J'en retire de beaux souvenirs d'une époque où j'étais très naïve et très ignorante des réalités politiques, des expériences qui avaient été faites ailleurs, des dangers de la censure, de la politique, quelle qu'elle soit, sur les expériences artistiques. J'en retire aussi un approfondissement de l'irréconciliabilité de la pratique politique de recherches scientifiques, philosophiques ou artistiques. Je ne parle pas d'une politique utopiste, telle qu'elle a pu être pensée par certains socialistes du siècle dernier, ou encore, par les philosophes de l'antiquité grecque, je parle de la politique telle que nous la connaissons partout.

V.I. Et vous avez trouvé nécessaire à un moment donné de sortir de ce type d'écriture et de ce type de recherche?

M.G. Pour reprendre l'expression de Pasolini : je ne renie pas ce que j'ai pensé ou écrit, mais je serais portée à "abjurer" certains de mes textes, certaines parties de mes textes qui, en fin de compte, contribuaient à cette mise en place des conditions d'une censure politique sur le déroulement d'une œuvre. J'ai appronfondi ça. Quand j'ai quitté Chroniques, j'ai remis un petit texte qui s'appelait: "La mort du texte en moi", texte qui n'a sans doute pas été tellement lu et compris parce que je me trouvais à dire que même dans ce lieu qui se croyait très pur, il y avait du pouvoir. II y en avait à voir, à analyser, à dénoncer, il y avait de la censure et comme ce n'était plus possible de le dire en ce lieu, je quittais; je partais en parlant d'écriture comme d'un lieu possible de non-pouvoir. Avec Blanchot, je parlerais aujourd'hui «d'impouvoir». Dans l'Écriture du désastre, cette problématique est merveilleusement pensée. C'est un peu pareil quand j'ai quitté mes fonctions dirigeantes au syndicat des professeurs de I'U.Q.A.M. Je l'ai fait aussi avec un long texte qui parlait du pouvoir tel que je l'avais vu se manifester, à gauche comme à droite. C'est un texte qui n'a pas été très bien reçu parce que je disais sans doute des choses qu'on ne voulait pas entendre. Qui, se prétendant à gauche, voudrait se faire dire que là aussi, on peut reproduire du pouvoir dans toutes ses manifestations? De façon générale, chez les intellectuels, le pouvoir se manifeste de manière plus subtile, insidieuse: il fonctionne davantage par les voies de la séduction et de la vénération. C'était une parole sur le pouvoir qui était à toute fin pratique irrecevable. Inadmissible.

V.I. Vous semblez amère. 
M.G. Pas du tout. Au contraire, j'ai beaucoup appris des rapports entre organisations politiques, discours et paroles politiques, discours et paroles critiques et créatrices. J'ai pu $\mathrm{y}$ approfondir aussi le lieu absolument intouchable et inviolable de l'écriture par le pouvoir, quel qu'il soit et accepter d'assumer la solitude de chaque écriture.

V.I. On vous invite, on vous cite, on vous consulte, on vous lit, comment recevez-vous cette reconnaissance du milieu intellectuel?

M.G. Comme je reconnais d'autres écrivains, il me fait toujours plaisir quand la reconnaissance se manifeste à mon égard. Comme disait Barthes: «Je ne crois pas qu'on écrive pour être reconnu, mais on écrit pour être aimé». Je ne crois pas que j'aie tant d'importance, d'influence auprès des femmes. J'ai très peu de lecteurs et de lectrices comme la plupart des écrivains qui pratiquent ce type d'écriture, pas toujours facile. On ne peut pas parler d'une très grande influence quand on a quelques lecteurs. L'écriture est une activité isolée, la lecture aussi. On prend connaissance d'un texte dans un isolement souvent total, dans le silence. Quand il se produit du bruit, de la cacophonie, je ne peux pas dire que ça me fasse plaisir. J'aime les reconnaissances qui se manifestent dans des paroles ou des lectures qui sont amoureuses de ce que j'ai écrit. Je n'éprouve pas le besoin d'une reconnaissance politique - j'ai l'impression que j'ai été au bout de ce fantasme-là. J'ai besoin d'amour, des signes de l'intelligence amoureuse, et ça, quand ça se manịeste, ça me fait plaisir. 